\title{
Assessment of Tumor-infiltrating Lymphocytes Predicts the Behavior of Early-stage Oral Tongue Cancer
}

\section{Heikkinen, llkka}

$2019-10$

Heikkinen , I, Bello , I O , Wahab , A , Hagström , J , Haglund , C , Coletta , R D , Nieminen , P , Makitie , A A , Salo , T , Leivo , I \& Almangush , A 2019 , ' Assessment of Tumor-infiltrating Lymphocytes Predicts the Behavior of Early-stage Oral Tongue Cancer ' , American Journal of Surgical Pathology, vol. 43 , no. 10 , pp. 1392-1396 . https://doi.org/10.1097/PAS.00000000000

http://hdl.handle.net/10138/324427

https://doi.org/10.1097/PAS.0000000000001323

cc_by_nc

acceptedVersion

Downloaded from Helda, University of Helsinki institutional repository.

This is an electronic reprint of the original article.

This reprint may differ from the original in pagination and typographic detail.

Please cite the original version. 


\section{Assessment of tumor-infiltrating lymphocytes predicts the behavior of early- stage oral tongue cancer}

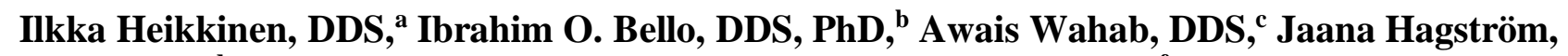
DDS, PhD,${ }^{\mathrm{d}}$ Caj Haglund, MD, PhD,${ }^{\mathrm{e}}$ Ricardo D. Coletta, DDS, PhD, ${ }^{\mathrm{f}}$ Pentti Nieminen, MSc, PhD, ${ }^{\mathrm{g}}$ Antti A. Mäkitie, MD, PhD, ${ }^{\mathrm{h}}$ Tuula Salo, DDS, PhD, ${ }^{\mathrm{i} *}$ Ilmo Leivo, MD, PhD, ${ }^{\mathrm{j} *}$ Alhadi Almangush, DDS, PhD $^{\mathrm{k} *}$

${ }^{a}$ Department of Pathology, University of Helsinki, Helsinki, Finland.

Department of Oral and Maxillofacial Diseases, University of Helsinki, Helsinki, Finland.

${ }^{\mathrm{b}}$ Department of Oral Medicine and Diagnostic Sciences, King Saud University College of Dentistry, Riyadh, Saudi Arabia.

Department of Pathology, University of Helsinki, Helsinki, Finland.

${ }^{\mathrm{c}}$ Department of Pathology, University of Helsinki, Helsinki, Finland. Department of Oral and Maxillofacial Diseases, University of Helsinki, Helsinki, Finland.

${ }^{\mathrm{d}}$ Department of Pathology, University of Helsinki, Helsinki, Finland.

Research Programs Unit, Translational Cancer Biology, University of Helsinki, Helsinki, Finland.

${ }^{\mathrm{e}}$ Research Programs Unit, Translational Cancer Biology, University of Helsinki, Helsinki, Finland. Department of Surgery, University of Helsinki and Helsinki University Hospital, Helsinki, Finland.

${ }_{\mathrm{f}}^{\mathrm{f}}$ Department of Oral Diagnosis, School of Dentistry, University of Campinas, Piracicaba, São Paulo, Brazil.

${ }^{\mathrm{g}}$ Medical Informatics and Data Analysis Research Group, University of Oulu, Oulu, Finland.

${ }^{\mathrm{h}}$ Department of Otorhinolaryngology - Head and Neck Surgery, University of Helsinki and Helsinki University Hospital, Helsinki, Finland.

Research Programme in Systems Oncology, Faculty of Medicine, University of Helsinki, Helsinki, Finland. Division of Ear, Nose and Throat Diseases, Department of Clinical Sciences, Intervention and Technology, Karolinska Institutet and Karolinska University Hospital, Stockholm, Sweden.

${ }^{\mathrm{i}}$ Department of Pathology, University of Helsinki, Helsinki, Finland.

Department of Oral and Maxillofacial Diseases, University of Helsinki, Helsinki, Finland.

Cancer and Translational Medicine Research Unit, Medical Research Center Oulu, University of Oulu and Oulu University Hospital, Oulu, Finland.

${ }^{\mathrm{j}}$ Institute of Biomedicine, Pathology, University of Turku, Turku, Finland.

${ }^{\mathrm{k}}$ Department of Pathology, University of Helsinki, Helsinki, Finland.

Research Programme in Systems Oncology, Faculty of Medicine, University of Helsinki, Helsinki, Finland. Institute of Biomedicine, Pathology, University of Turku, Turku, Finland.

Institute of Dentistry, University of Misurata, Misurata, Libya.

*equal contribution.

Corresponding author: Alhadi Almangush, DDS, $\mathrm{PhD}$

Department of Pathology, University of Helsinki, Helsinki, Finland

Haartmaninkatu 3 (P.O. Box 21), FIN-00014 University of Helsinki

e-mail: alhadi.almangush@helsinki.fi or alhadi.almangush@gmail.com

Telephone number: 00358452044668 
1 Funding: This study funded by Turku University Hospital Fund, Helsinki University Hospital

2 Research Fund, the Finnish Dental Society, the Rauha Ahokas Foundation, K. Albin Johanssons 3 Foundation, the Sigrid Juselius Foundation, the Finnish Cancer Society, Finska Läkaresällskapet, and 4 the Maritza and Reino Salonen Foundation.

5

6 Conflict of interest: None declared.

7

8

9

10

11

12

13

14

15

16

17

18

19

20

21

22

23 


\section{Abstract}

2 Tumor-infiltrating lymphocytes (TILs) have shown a promising prognostic value in many epithelial

3 cancers. We sought to assess the prognostic value of TILs in a multicenter cohort of early oral tongue

4 squamous cell carcinoma (OTSCC). The percentage of TILs was assessed on the surgical resection

5 slides stained with hematoxylin and eosin (HE). The assessment of TILs was performed in the stromal

6 compartment and in the intra-epithelial compartment (at the invasive front and at the center of the

7 tumor). We followed the method that was described recently by the International Immuno-Oncology

8 Biomarker Working Group for the assessment of TILs. A total of 308 cases from the five Finnish

9 university hospitals and from A.C. Camargo Cancer Center, São Paulo, Brazil were included. We

10 found a promising prognostic value for stromal TILs at the invasive front in the multivariable analysis

11 with a hazard ratio of $2.61(95 \%$ CI $1.77-3.83 ; P<0.001)$ for overall survival, 1.99 (95\% CI 1.07-3.69;

$12 P=0.040)$ for disease-specific survival, and $1.94(95 \% \mathrm{CI} 1.14-3.29 ; P=0.020)$ for disease-free

13 survival. In conclusion, evaluation of TILs is simple and can aid in identifying the high-risk cases of

early OTSCC. The method introduced by the International Immuno-Oncology Biomarker Working

Group can be used for standardized determination of TILs in early OTSCC.

Keywords: Oral tongue squamous cell carcinoma (OTSCC); Tumor-infiltrating lymphocytes (TILs); 
2 Oral tongue squamous cell carcinoma (OTSCC) is associated with worse survival compared to SCC

3 of other sites of the oral cavity ${ }^{1}$. The current prognostic biomarkers of early-stage OTSCC do not 4 provide optimal risk stratification ${ }^{2}$. Therefore, searching for "informative, simple and reliable" 5 biomarkers is of great importance, since those biomarkers will form a cornerstone for individualized

6 treatment. The role of tumor microenvironment in cancer development and prognosis has evoked 7 increasing interest among cancer researchers in recent years. Immune cells comprise one component 8 of the tumor microenvironment and the immune infiltrates associating with tumors have been studied $9 \quad$ widely ${ }^{3,4}$.

Pathological prognostication of clinical behavior is usually based on specific histopathologic

features like tumor grade, pattern of invasion and perineural invasion. Recent research indicates that the immune response is a key player during cancer progression ${ }^{5}$. Moreover, an increasing number of evidence shows immune response to be a viable prognostic marker and, in many cases, even superior to the TNM classification ${ }^{6}$. Therefore, it is suggested that tumor-infiltrating lymphocytes (TILs) or similar immunoscores should be included in standard pathological classifications ${ }^{6}$. However, the routine pathology report does not evaluate the immune response in OTSCC. Therefore, it is of clinical importance to find histopathologic criteria that can effectively assess immune response using the routine hematoxylin and eosin (HE)-stained slides.

The International Immuno-Oncology Biomarker Working Group ${ }^{7,8}$ has recently introduced guidelines for assessment of TILs in solid tumors using HE-stained sections ${ }^{7}$. Growing evidence highlights the significance of this new method in evaluating the TILs as a determining factor of tumor biology with special focus on their prognostic/predictive value ${ }^{8}$. Although the histological assessment of TILs in HE-stained samples does not reveal the different subpopulations of lymphocytes, it may yet prove to be a useful biomarker for assessing tumor behavior. Among the several advantages of this method is its cost-effectiveness, no requirement of expensive or specific 
1 tools or antibodies, and easy incorporation into standard pathology report. In this study, we

2 investigated the significance of TILs as a potential prognostic marker in early OTSCC using a multi-

3 institutional cohort that we have used in our previous study ${ }^{9}$.

\section{$5 \quad$ Material and Methods}

6 Patients: This study included 308 patients (163 men and 145 women) treated for early OTSCC at one of the five Finnish University Hospitals (Helsinki, Turku, Tampere, Oulu, and Kuopio) or at the A.C. Camargo Cancer Center, São Paulo, Brazil. The primary treatment of patients in our cohort was surgical resection. The use of patient samples and the data enquiry were approved by the abovementioned University hospitals, by the Brazilian Human Research Ethics Committee and by the Finnish National Supervisory Authority for Welfare and Health (VALVIRA).

Scoring of TILs: A training session was arranged to familiarize the observers with the scoring criteria. We evaluated TILs according to a scoring method introduced recently by the International Immuno-Oncology Biomarkers Working Group ${ }^{7}$. In brief, intra-tumoral TILs were scored as the percentage of tumor islands occupied by lymphocytes; and stromal TILs were defined as the percentage of stroma occupied by lymphocytes. Any stromal area that did not relate directly to the tumor was not included in the estimation of TILs. Moreover, areas of fibrosis or central necrosis were not included in the assessment of TILs ${ }^{7}$. The percentage of TILs was assessed in two regions of each sample (at the invasive front and at the central part of the tumor). Assessment of TILs was made in two compartments, the intra-tumoral (i.e. intra-epithelial) and the stromal compartment. Tumor slides were visually scanned by light microscope, and the percentage of TILs at the invasive front was estimated separately for the intra-tumoral part and for the stromal part. The same scoring method was used to assess TILs in the central region of the tumor. The TILs working group guidelines ${ }^{7,10}$ has pointed out "Do not focus on hot spots". Therefore, the average of TILs in the stromal area was considered when reporting stromal TILs. Similarly, the average of TILs in the tumor area was 
1 considered when reporting intra-tumoral TILs. The percentage of TILs was assessed semi-

2 quantitatively as an incremental parameter (e.g. 5\%, 10\%, 20\%, 30\% ...) as previously described ${ }^{7,10}$.

3 For example, 50\% stromal TILs indicates that half of the stromal area is occupied by infiltrating

4 lymphocytes. The sample was scanned at low magnification (with 5-10× objectives), then the average

5 percentage of TILs across microscopic fields was estimated at higher magnification (with 20-40x

6 objectives). At least five fields were evaluated to assess the average of TILs. We used full untrimmed

7 sections from the resected tumors as has been recommended ${ }^{10}$. At least one representative section

$8 \quad(4-5 \mu \mathrm{m})$ was available for each case included in our study. Low-quality tumor sections (e.g. those

9 without tumor-stroma interface) were excluded from our cohort.

10 The scoring of TILs was performed using HE-stained slides (Fig. 1). Evaluation of TILs was 11 conducted by an independent researcher (IH); all sections were reviewed by a senior researcher (IB), 12 and then we calculated the degree of agreement between the observers.

13 Data analysis: Cohens's kappa was used to estimate the inter-rater reliability between the observers 14 in classifying the tumors into low and high TILs. Cross-tabulation and Chi-square test was also 15 applied to evaluate the associations between TILs and age, gender, cTNM, WHO grade and PNI.

16 Univariable and multivariable analyses were conducted to evaluate the associations between 17 explanatory variables and the overall survival (OS), disease-specific survival (DSS) and disease-free survival (DFS) using the Cox regression model ${ }^{11}$. In multivariable analysis, only those variables that appeared statistically significant in the univariable analysis were included for the estimated models. Therefore, TILs (low vs high) was analysed as the main explanatory variable; and age, stage, gender, WHO grade and perineural invasion (PNI) were used as covariates in the multivariable models. 22 Hazard ratios (HR) and 95\% confidence intervals (95\% CI) were reported for both univariable and 23 multivariable analyses. The proportional hazards assumptions of Cox regression were met by the 24 data. Kaplan-Meier survival curves were used to describe the OS, DSS and DFS based on TILs. We used log-rank test to evaluate the statistical significance between the survival curves. The statistical 
analyses were performed using IBM SPSS Statistics (version 24) and MedCalc (version 18). A $P$ value of $<0.05$ (two sided) was considered as statistically significant.

\section{Results}

A total of 308 patients clinically classified as T1N0M0 (122 cases) or T2N0M0 (186 cases) were included in this study.

The presence of TILs in the stromal part (i.e. sTILs) ranged from $1 \%$ to $95 \%$, and in the intratumoral/intra-epithelial part (i.e. iTILs) ranged from $0 \%$ to $25 \%$. Different cutoff points $(5 \%, 10 \%$, $20 \%$...etc) were applied to stratify the tumors as containing low or high TILs. The cutoff point that was clinically most relevant was found to be $20 \%$ (i.e. low TILs $\leq 20 \%$; high TILs $>20 \%$ ) at the invasive front. Fifty-one tumors (16.6\%) had low TILs and 257 tumors (83.4\%) had high TILs. The relationship between sTIL and the recorded clinicopathologic features is summarized in Table 1 . There was a statistically significant association between sTILs and iTILs $(P=0.021)$. No association was found between sTILs and age, gender, cTNM stage, or WHO grade $(P>0.05)$. sTILs showed an association with perineural invasion $(P=0.025)$. We found a moderate agreement between the observers in classifying the tumors into low TILs or high TILs (Cohen's kappa $=0.64$ ).

In the univariable analysis (Table 2$)$, low sTILs $(\leq 20 \%)$ at the invasive front was found as an adverse prognostic factor for OS (HR 2.46, 95\% CI 1.70-3.57, $P<0.001$ ), associated with high cancer mortality (DSS HR 2.02, 95\%CI 1.11-3.66, $P=0.021$ ) and worse DFS (HR 1.93, 95\%CI 1.16-3.22, $P=0.011$ ). While age of the patient was also associated with OS, DSS and DFS (Table 2), the remaining variables (gender, stage, grade and perineural invasion) were insignificant to be considered as factors for prognostication. These significant relationships between sTILs and OS, DSS or DFS are exemplified in the Kaplan-Meier curves (Fig 2 A, B, and C). In multivariable analysis (Table 2), sTILs showed a promising prognostic value for prediction of OS (HR 2.61, 95\%CI 1.77-3.83; $P<$ 0.001), DSS (HR 1.99, 95\%CI 1.07-3.69; $P=0.040)$, and DFS (HR 1.94, 1.14-3.29; $P=0.020)$. 
Intra-tumoral infiltrating lymphocytes did not show a significant prognostic value in univariable analyses of OS (HR 1.14, 95\%CI 0.76-1.68; $P=0.51)$, DSS (HR 1.06, 95\%CI 0.57-1.94; $P=0.863)$ or DFS (HR 1.06, 95\%CI 0.63-1.78; $P=0.834)$. The insignificant prognostic values of

4 intra-tumoral infiltrating lymphocytes were confirmed in the multivariable analyses of OS (HR 1.05, $5 \quad 0.71-1.55 ; P=0.818)$, DSS (HR $1.19,95 \%$ CI $0.63-2.22 ; P=0.594)$ and DFS (HR 1.09, 95\%CI 0.64$6 \quad 1.85 ; P=0.761)$.

\section{Discussion}

9 For clinicians, it is a dilemma to identify those cases of early OTSCC that will potentially have an aggressive behavior and would thus benefit from chemoradiotherapy and/or neck dissection. Many factors will determine tumor behavior and risk of poor survival. Of these factors, immune response has been shown to possess a fundamental role in identifying aggressive tumors ${ }^{6}$. In this study, we used the HE-stained slides from surgical specimens to evaluate TILs in a multicenter series of early OTSCC and we found that tumors with low percentage of stromal TILs $(\leq 20 \%)$ at the invasive front have a significantly poor survival (overall, disease-specific and disease-free).

As the current method of evaluating TILs was based on the use of routine H-E slides, it will be possible to assess them routinely in the daily practice of pathologists and will provide a better histopathologic prognostication based on immune response. Such prognostication may be useful for clinical decision-making including, for example, selecting early-stage OTSCC cases for multimodality treatments. Recent research highlighted the role of the immune cells in modulating

21 cancer invasion and metastasis ${ }^{12}$. Therefore, the immune response is assumed to influence the clinical 22 behavior of tumors. In fact, tumors of the same clinical stage and/or same histopathologic grade may 23 have extremely variable immune responses ${ }^{6}$. Thus, the immunological heterogeneity of early OTSCC can be utilized to classify cases into low-risk and high-risk groups. 
Recently, we have systematically reviewed the prognostic value of all immune checkpoints

2 that have been studied in oral squamous cell carcinoma ${ }^{13}$. We noted that the currently available body

3 of evidence still require further research and none of the studied immune biomarkers can be approved

4 for prognostication in daily practice ${ }^{13}$. In addition, previous studies have used a specific

5 immunohistochemical staining which is not routinely ordered by the pathologists. In our previous

6 research, we evaluated the lymphocytic host response (LHR) according to criteria described in the

7 histologic risk score ${ }^{14}$ and we reported a low prognostic value of that criteria for prognostication of

8 early OTSCC ${ }^{15}$. Noteworthy, LHR was divided into three categories (strong, intermediate or weak)

9 without quantitative measurement of the immune response. On the other hand, the International

Immuno-Oncology Biomarkers Working Group has indicated that assessment of TILs should be done as a continuous semiquantitative variable and did not determine risk threshold between high and low TILs in early OTSCC ${ }^{7}$.

Currently, a method for overall assessment of TILs (i.e. using HE-stained slides) after neoadjuvant treatment of OTSCC has not yet been established. In cases with neoadjuvant therapy determining the area of the residual tumor to be used for evaluation of TILs requires further research ${ }^{16}$. A small and/or superficial preoperative biopsy of oral cancer might also be a problematic as it may not include the most important area for assessment of TILs (i.e. within the borders of the invasive tumor). Such limitation of biopsies of oral cancer has been noted during the assessment of other prognostic features ${ }^{17}$. Therefore, it is necessary to consider having a representative sample that is deep enough including the invasive front for the assessment of TILs.

Recently, there is a worldwide research effort to standardize determination of TILs. Many studies have reported that a strong lymphocytic infiltration associates with favorable clinical outcome

23 of different tumors including those of head and neck ${ }^{18-20}$. Such favorable outcome could be due to 24 the destruction of cancer cells and anti-tumor effect of the immune response ${ }^{21}$. In conclusion, 25 assessment of TILs has a reliable prognostic value in early OTSCC. The method introduced recently 
by the International Immuno-Oncology Biomarkers Working Group is simple, cost-effective and can be easily included in the pathology report. To the best of our knowledge, this is the first multicenter study on early OTSCC applying the new criteria for evaluation of TILs. Our finding on TILs can be a key step towards the routine measurement of immune-response in early OTSCC and that could enhance the personalized treatment approach by classifying risk groups based on immune response.

(6)

\section{References}

1. Farhood Z, Simpson M, Ward GM, et al. Does anatomic subsite influence oral cavity cancer mortality? A SEER database analysis. Laryngoscope. 2018.

2. Almangush A, Heikkinen I, Makitie AA, et al. Prognostic biomarkers for oral tongue squamous cell carcinoma: a systematic review and meta-analysis. Br J Cancer. 2017;117:856-866.

3. Wang M, Zhao J, Zhang L, et al. Role of tumor microenvironment in tumorigenesis. J Cancer. 2017;8:761-773.

4. Giraldo NA, Sanchez-Salas R, Peske JD, et al. The clinical role of the TME in solid cancer. Br J Cancer. 2019;120:45-53.

5. Pandya PH, Murray ME, Pollok KE, et al. The Immune System in Cancer Pathogenesis: Potential Therapeutic Approaches. J Immunol Res. 2016;2016:4273943.

6. Galon J, Pages F, Marincola FM, et al. Cancer classification using the Immunoscore: a worldwide task force. J Transl Med. 2012;10:205.

7. Hendry S, Salgado R, Gevaert T, et al. Assessing Tumor-Infiltrating Lymphocytes in Solid Tumors: A Practical Review for Pathologists and Proposal for a Standardized Method from the International Immuno-Oncology Biomarkers Working Group: Part 2: TILs in Melanoma, Gastrointestinal Tract Carcinomas, Non-Small Cell Lung Carcinoma and Mesothelioma, Endometrial and Ovarian Carcinomas, Squamous Cell Carcinoma of the Head and Neck, Genitourinary Carcinomas, and Primary Brain Tumors. Adv Anat Pathol. 2017;24:311-335.

8. Dieci MV, Radosevic-Robin N, Fineberg S, et al. Update on tumor-infiltrating lymphocytes (TILs) in breast cancer, including recommendations to assess TILs in residual disease after neoadjuvant therapy and in carcinoma in situ: A report of the International Immuno-Oncology Biomarker Working Group on Breast Cancer. Semin Cancer Biol. 2018;52:16-25.

9. Almangush A, Heikkinen I, Bakhti N, et al. Prognostic impact of tumour-stroma ratio in earlystage oral tongue cancers. Histopathology. 2018;72:1128-1135.

10. Salgado R, Denkert C, Demaria S, et al. The evaluation of tumor-infiltrating lymphocytes (TILs) in breast cancer: recommendations by an International TILs Working Group 2014. Ann Oncol. 2015;26:259-271.

11. Kleinbaum DG, Klein M. Survival Analysis: A Self-Learning Text, Third Edition. 2012.

12. Kitamura T, Qian BZ, Pollard JW. Immune cell promotion of metastasis. Nat Rev Immunol. 2015;15:73-86.

13. Sievilainen M, Almahmoudi R, Al-Samadi A, et al. The Prognostic Value of Immune Checkpoints in Oral Squamous Cell Carcinoma. Oral Dis. 2018. 
1 14. Brandwein-Gensler M, Smith RV, Wang B, et al. Validation of the histologic risk model in a 2 new cohort of patients with head and neck squamous cell carcinoma. Am J Surg Pathol. 2010;34:6763688.

4 15. Almangush A, Bello IO, Keski-Santti H, et al. Depth of invasion, tumor budding, and worst 5 pattern of invasion: prognostic indicators in early-stage oral tongue cancer. Head Neck. 2014;36:8116818.

7 16. Kurozumi S, Inoue $\mathrm{K}$, Matsumoto $\mathrm{H}$, et al. Prognostic utility of tumor-infiltrating 8 lymphocytes in residual tumor after neoadjuvant chemotherapy with trastuzumab for HER2-positive 9 breast cancer. Sci Rep. 2019;9:1583.

10 17. Dhanda J, Uppal N, Chowlia H, et al. Features and prognostic utility of biopsy in oral 11 squamous cell carcinoma. Head Neck. 2016;38 Suppl 1:E1857-1862.

12 18. Denkert C, von Minckwitz G, Darb-Esfahani S, et al. Tumour-infiltrating lymphocytes and 13 prognosis in different subtypes of breast cancer: a pooled analysis of 3771 patients treated with 14 neoadjuvant therapy. Lancet Oncol. 2018;19:40-50.

15 19. Rakaee M, Kilvaer TK, Dalen SM, et al. Evaluation of tumor-infiltrating lymphocytes using 16 routine $\mathrm{H} \& \mathrm{E}$ slides predicts patient survival in resected non-small cell lung cancer. Hum Pathol. 17 2018;79:188-198.

18 20. Almangush A, Ruuskanen M, Hagstrom J, et al. Tumor-infiltrating lymphocytes associate with outcome in non-endemic nasopharyngeal carcinoma: a multicenter study. Hum Pathol. 2018. 21. Fridman WH, Pages F, Sautes-Fridman C, et al. The immune contexture in human tumours: impact on clinical outcome. Nat Rev Cancer. 2012;12:298-306. 


\section{Figure legend}

2 Figure 1: Tumor-infiltrating lymphocytes (TILs) were evaluated on full sections of early OTSCC

3 stained with hematoxylin and Eosin. Representative samples classified as low $(\leq 20 \%)$ stromal TILs 4 (A); and high (> 20\%) stromal TILs (B).

5

$6 \quad$ Figure 2: Kaplan-Meier curves for stromal TILs with OS (A), DSS (B), and DFS (C).

7 TILs: Tumor-infiltrating lymphocytes; OS: Overall survival; DSS: Disease-specific survival; DFS:

8 Disease-free survival.

9 


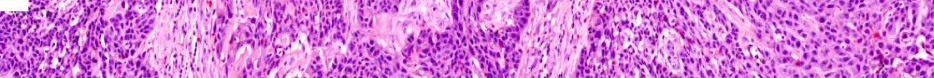
(26)

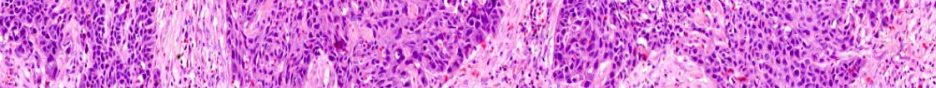

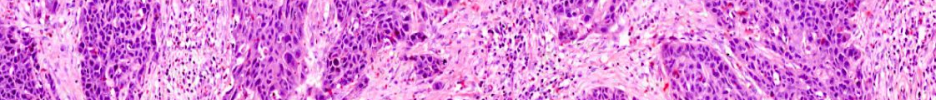

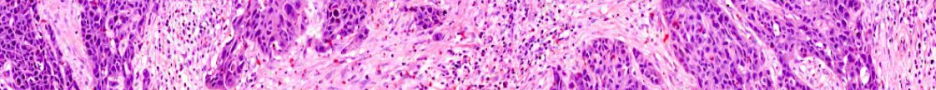

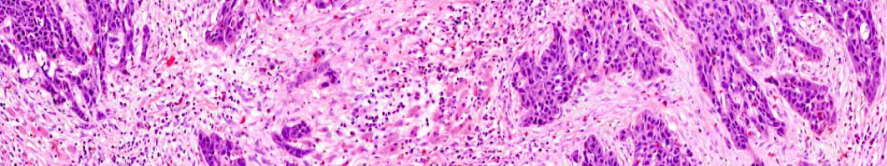

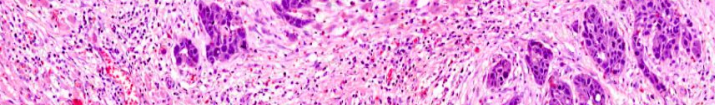

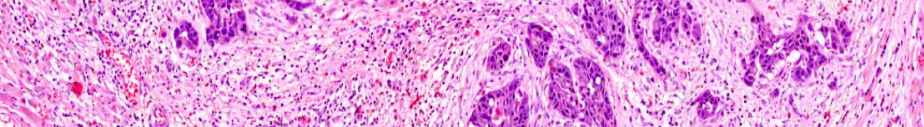

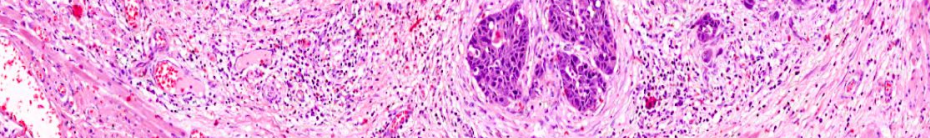

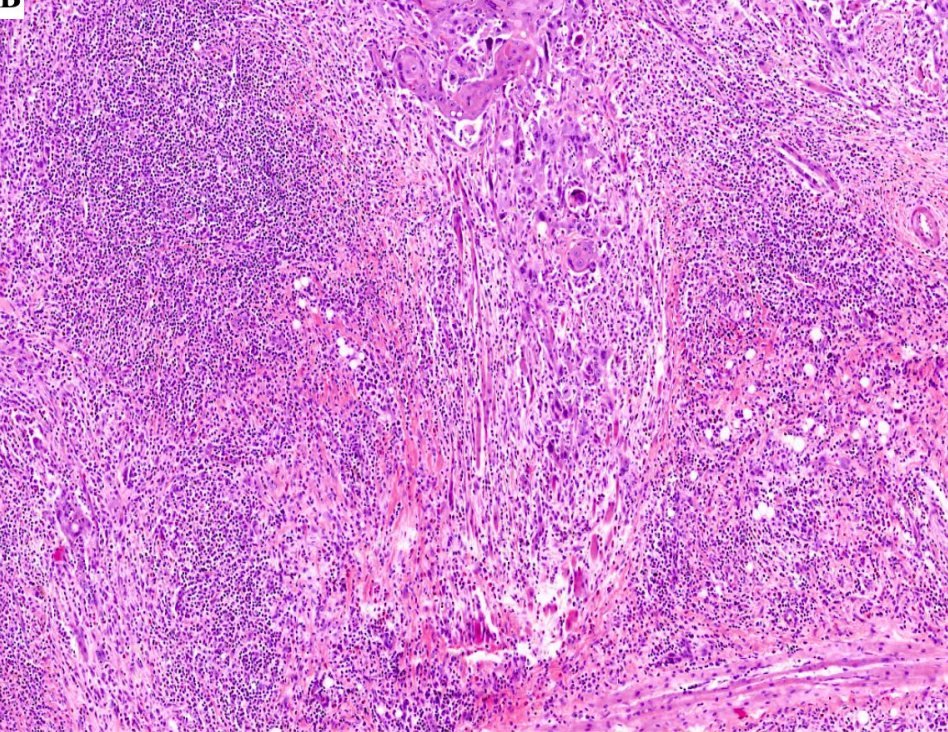

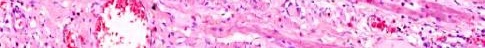


Table 1: Relationship between tumor-infiltrating lymphocytes (TILs) and clinicopathologic characteristics of 308 cases treated for early oral tongue cancer.

\begin{tabular}{|c|c|c|c|c|}
\hline \multirow[t]{2}{*}{ Variable } & Total & High sTILs & Low sTILs & $\begin{array}{l}P \text { value of } \\
\text { chi-square } \\
\text { test }\end{array}$ \\
\hline & Total, $\mathrm{N}=308$ & $\begin{array}{l}\text { Number (\%) } \\
257(83.4) \\
\end{array}$ & $\begin{array}{l}\text { Number }(\%) \\
51(16.6)\end{array}$ & \\
\hline Age & & & & 0.535 \\
\hline$\leq 60$ & 129 & $110(85.3)$ & $19(14.7)$ & \\
\hline$>60$ & 179 & $147(82.1)$ & $32(17.9)$ & \\
\hline Gender & & & & 0.363 \\
\hline Male & 163 & $133(81.6)$ & $30(18.4)$ & \\
\hline Female & 145 & $124(85.5)$ & $21(14.5)$ & \\
\hline cTNM stage* & & & & 0.188 \\
\hline T1N0M0 & 122 & $106(86.9)$ & $16(13.1)$ & \\
\hline T2N0M0 & 186 & $151(81.2)$ & $35(18.8)$ & \\
\hline Grade (WHO) & & & & 0.267 \\
\hline Well differentiated & 104 & $89(85.6)$ & $15(14.4)$ & \\
\hline Moderately differentiated & 129 & $110(85.3)$ & $19(14.7)$ & \\
\hline Poorly differentiated & 75 & $58(77.3)$ & $17(22.7)$ & \\
\hline PNI & & & & 0.025 \\
\hline Absent & 267 & $228(85.4)$ & $39(14.6)$ & \\
\hline Present & 41 & $29(70.7)$ & $12(29.3)$ & \\
\hline iTILs & & & & 0.021 \\
\hline High & 245 & $198(80.8)$ & $47(19.2)$ & \\
\hline Low & 63 & $59(93.7)$ & $4(6.3)$ & \\
\hline
\end{tabular}

* cTNM stage refers to the 7th edition of AJCC staging manual as our cases were treated before 2017. Abbreviations: iTILs: intra-tumoral TILs; sTILs: stromal TILs. 
Table 2: Univariable and multivariable analyses of 308 cases treated for early OTSCC. The analyses include overall survival (OS), disease-specific survival (DSS), and disease-free survival (DFS) for tumorinfiltrating lymphocytes (TILs) and the adjusting variables.

\begin{tabular}{|c|c|c|c|}
\hline \multirow[t]{3}{*}{ Variable } & \multicolumn{3}{|c|}{ Univariable analysis } \\
\hline & OS & DSS & DFS \\
\hline & HR (95\% CI) & HR (95\% CI) & HR (95\% CI) \\
\hline \multicolumn{4}{|l|}{ Age } \\
\hline$\leq 60$ & 1 & 1 & 1 \\
\hline \multirow[t]{2}{*}{$>60$} & $2.17(1.55-3.03)$ & $1.89(1.11-3.21)$ & $1.76(1.13-2.75)$ \\
\hline & $P<0.001$ & $P=0.020$ & $P=0.013$ \\
\hline \multicolumn{4}{|l|}{ Gender } \\
\hline Male & 1 & 1 & 1 \\
\hline \multirow[t]{2}{*}{ Female } & $0.79(0.57-1.08)$ & $1.20(0.73-1.97)$ & $1.10(0.72-1.68)$ \\
\hline & $P=0.134$ & $P=0.464$ & $P=0.651$ \\
\hline \multicolumn{4}{|l|}{ Stage } \\
\hline T1N0M0 & 1 & 1 & 1 \\
\hline \multirow[t]{2}{*}{ T2NOM0 } & $1.26(0.90-1.76)$ & $1.47(0.86-2.51)$ & $0.89(0.58-1.37)$ \\
\hline & $P=0.177$ & $P=0.164$ & $P=0.603$ \\
\hline \multicolumn{4}{|l|}{ Grade (WHO) } \\
\hline Well differentiated & 1 & 1 & 1 \\
\hline \multirow[t]{2}{*}{ Moderately differentiated } & $1.36(0.95-1.96)$ & $1.70(093-3.11)$ & $1.08(0.66-1.77)$ \\
\hline & $P=0.094$ & $P=0.085$ & $P=0.748$ \\
\hline \multirow[t]{2}{*}{ Poorly differentiated } & $1.10(0.71-1.69)$ & $1.58(0.79-3.16)$ & $1.25(0.72-2.16)$ \\
\hline & $P=0.655$ & $P=0.196$ & $P=0.435$ \\
\hline \multicolumn{4}{|l|}{ PNI } \\
\hline Absent & 1 & 1 & 1 \\
\hline \multirow[t]{2}{*}{ Present } & $1.32(0.86-2.01)$ & $1.32(0.67-2.59)$ & $1.38(0.78-2.44)$ \\
\hline & $P=0.204$ & $P=0.424$ & $P=0.270$ \\
\hline \multicolumn{4}{|l|}{ iTILs } \\
\hline High & 1 & 1 & 1 \\
\hline \multirow[t]{2}{*}{ Low } & $1.14(0.76-1.68)$ & $1.06(0.57-1.94)$ & $1.06(0.63-1.78)$ \\
\hline & $P=0.51$ & $P=0.863$ & $P=0.834$ \\
\hline \multicolumn{4}{|l|}{ sTILs } \\
\hline High & 1 & 1 & 1 \\
\hline \multirow[t]{2}{*}{ Low } & $2.46(1.70-3.57)$ & $2.02(1.11-3.66)$ & $1.93(1.16-3.22)$ \\
\hline & $P<0.001$ & $P=0.021$ & $P=0.011$ \\
\hline \multicolumn{4}{|c|}{ Multivariable analysis } \\
\hline \multicolumn{4}{|c|}{ 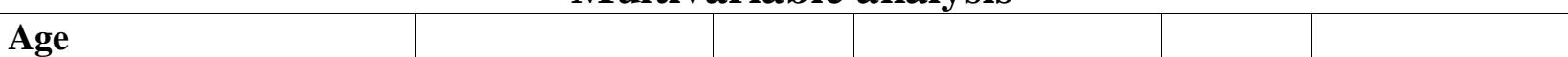 } \\
\hline$\leq 60$ & 1 & 1 & 1 \\
\hline \multirow[t]{2}{*}{$>60$} & $2.48(1.74-3.52)$ & $2.05(1.18-3.57)$ & $1.85(1.16-2.94)$ \\
\hline & $P=0.001$ & $P=0.009$ & $P=0.008$ \\
\hline \multicolumn{4}{|l|}{ sTILs } \\
\hline High & 1 & 1 & 1 \\
\hline \multirow[t]{2}{*}{ Low } & $2.61(1.77-3.83)$ & $1.99(1.07-3.69)$ & $1.94(1.14-3.29)$ \\
\hline & $P<0.001$ & $P=0.040$ & $P=0.020$ \\
\hline
\end{tabular}

Multivariable models were adjusted fort gender, stage, grade, and PNI.

iTILs: intra-tumoral TILs; sTILs: stromal TILs. 